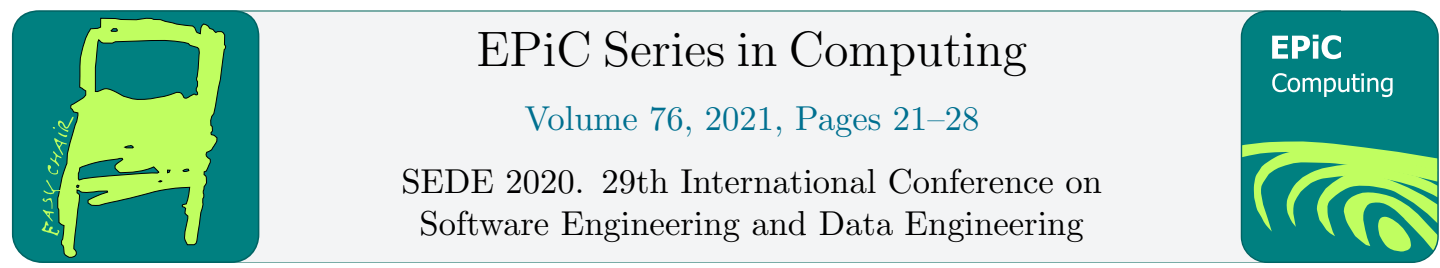

\title{
Cognitive Informatics in Emergency Disaster Management Systems
}

\author{
Ajay Bandi and Aziz Fellah \\ Northwest Missouri State University, Maryville, Missouri, U.S.A. \\ \{ajay, afellah\}@nwmissouri.edu
}

\begin{abstract}
In this research, we investigated the dynamic assignment of resources in emergency and disaster management systems that assists rescuers and responding agencies in effective real-time coordination. We also proposed a communication framework architecture, a common operating picture that keeps all communication activities among various stakeholders and agencies to manage emergency and disaster responses. This spectrum of activities is achieved through a comprehensive analytical emergency disaster management system that fetches locations using Google APIs geospatial data and infrastructures such as Google Maps. Also, we conducted usability testing by applying cognitive informatics principles to our emergency model. Such a model provides several services across several types of disasters and various locations such as monitoring emergencies and disasters, describing incidents, performing triage, accessing databases, analyzing the specific need of rescuers, and providing assistance within the professional role and jurisdiction status of every stakeholder member. We also found a few design issues in selecting the type of the stakeholder, limiting the data accesses to a few stakeholders, and filtering the historical data by triage codes.
\end{abstract}

\section{Introduction}

There are challenges in the world's economy in recent times due to the increase in natural and human-made disasters. According to the World Bank [10], the impact of natural disasters results in nearly $\$ 520$ billion of lost consumption annually. Furthermore, these disasters force almost 26 million people into poverty each year. Effective disaster preparedness and response systems are essential for reducing short-term impacts on public safety and the economy and ensuring rapid recovery from disasters. To accomplish this, a robust and intelligent Emergency Disaster Management System (EDMS) software is necessary.

Emergency informatics is an exploration of acquiring, analyzing, and visualizing the disasters' data for making the right decision to save the lives of victims [8]. Wang defined Cognitive Informatics "(CI) is an interdisciplinary research area that encompasses informatics, computer science, software engineering, mathematics, knowledge theory, cognition science, neurobiology, psychology, and physiology." [11] Researchers [8] identified the need for computing is essential in all the four phases (prevention, preparedness, response, and recovery) of disaster management. 
However, there are computing challenges such as scalability and decision-making support tools in extreme conditions [8].

Due to physical and emotional stress at the disaster location, responders experience physiological and cognitive workload in catastrophic situations. It is a challenge for the responders to perceive and process the information for reasoning and judging whether they are doing it right or wrong in a limited time [8]. Also, communicating with appropriate stakeholders takes a considerable amount of time in rescuing victims. There is a need to capture and describe the cognitive aspects of the responders' domain knowledge work in problem-solving and decision making. There exist several websites, and mobile apps [3] for managing disasters that recognize the importance of designing user interfaces and the usability of life-critical applications. However, this software lacks the processes of cognitive nature in the real world. The interdisciplinary area, cognitive informatics [11], facilitates investigating the human cognitive processes of responders and then applying computer science and information systems knowledge to automate for decision making and improve human-computer interfaces and the usability of the software.

Our contribution of this research is two-fold. 1) Dynamically assign the necessary and sufficient resources to rescue the victims. 2) Propose the Common Operating Picture (COP) with an interactive dashboard - COP enables the situational awareness of the disaster among different stakeholders. To achieve these, we developed the Community Emergency Response Team (CERT) application and conducting systematic usability testing by applying CI principles [11]. We extracted the working knowledge of the rescue teams using a think-aloud process while performing usability testing tasks [5]. This helps to know the thought process of the user and to identify the design issues. The rest of the paper is organized as follows. Section 2 discusses the related studies. Section 3 describes our comprehensive analytical emergency disaster management system's methodology for storing the historical data, assigning the resources. Section 4 describes the case study, Section 5, highlights the results, and Section 6 presents the conclusion and future research.

\section{Related Studies}

Researchers Yang et al. [12] designed a visualization tool called PhaseVis. They analyzed the Hurricane Issac tweets to investigate how the emergency disaster management phases are functioned effectively. They used SVM, Naïve Bayes, and 10-fold cross-validation machine learning algorithms. In their research, Kubicek and Stanek [6], discussed the dynamic visualization of emergency management by considering the various stakeholders, including local body reliefs of the Czech Republic. Bandi et al. designed the EDMS using design patterns [2], focused on the design issues of converting websites into mobile sites and apps [3], conducted systematic usability testing for websites [5] and mobile apps [4]. Zagorecki et al. [13] investigated the concept of executive dashboards. However, these studies did not focus entirely on cognitive informatics.

Researchers Mastrian and McGonigle [7] identified the need for cognitive informatics for nursing technology design. According to Pacific Northwest National Library [9], the goal of research in technology is to overcome the cognitive issues of memory overload, the ability to learn and comprehend, and the ability to make decisions.

However, these studies in the existing literature do not apply cognitive principles in emergency disaster management. We attempted to use the cognitive principles aspects of software engineering and human-computer interaction to design the common operating picture for various stakeholders and the resource assignment to the rescuers to reduce the cognitive workload. 


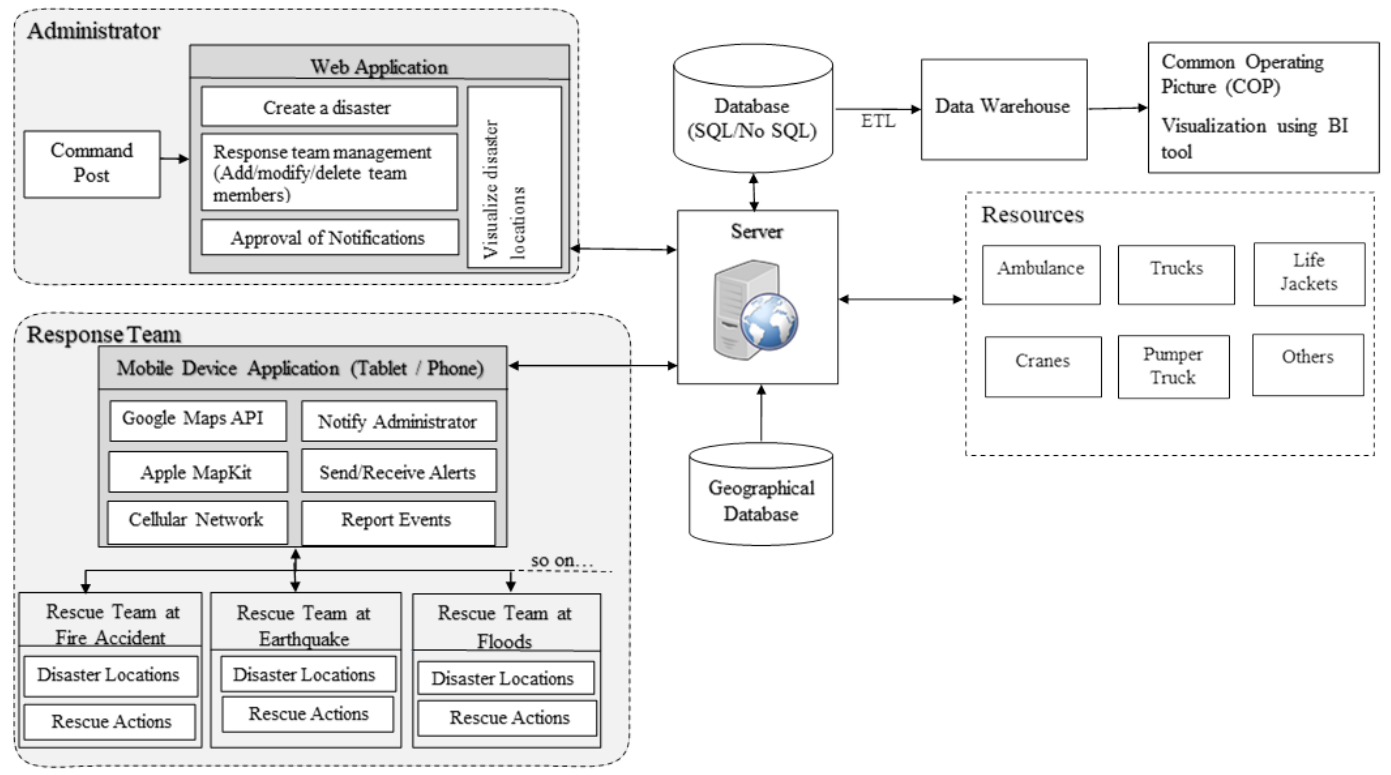

Figure 1: Comprehensive Analytical EDMS

\section{Methodology}

Our idea is to build on the EDMS that was proposed in our previous study [4]. Studies show that visual information plays a vital role in communicating and prioritize tasks. Our previous system was able to identify the rescue teams' location and visually disclose that information on maps. Also, other features include communication between teams, within the groups, and administrators were also implemented. For example, let us consider an earthquake disaster; the administrator assigned rescue teams to save the victims and property at the incident location. The organizations assess the people's loss and property damage and are in constant communication with the administrators through the mobile app. The administrator can visualize the team's location and guide them based on the information they receive from various sources. However, the system lacks the dynamic assigning of resources and the common operating picture to communicate and visualize by multiple stakeholders.

The architecture of the comprehensive analytical EDMS is shown in Fig. 1. The newly introduced features are automatically assigning the resources and visualizing the common operating picture. In continuation of the above example, based on the input provided by the rescue teams, the administrators would automatically assign the resources, such as ambulances, water trucks, aerial trucks, etc. automatically using the web application. These tasks reduce a significant amount of time in the process of rescuing. To achieve this, we used the principles of CI, to extract the knowledge and mental models of the rescuers by usability testing [5]. We used a think-aloud process to observe the design issues faced by the users. The details of the case study are given in the next section.

The new system's enhancements are to identify and assign the resources that help to rescue victims and to communicate different stakeholders through COP using a dynamic dashboard. The resources are fetched by using Google APIs and assigned by the administrators. The operational database is converted to a data warehouse to store the historical data using the 


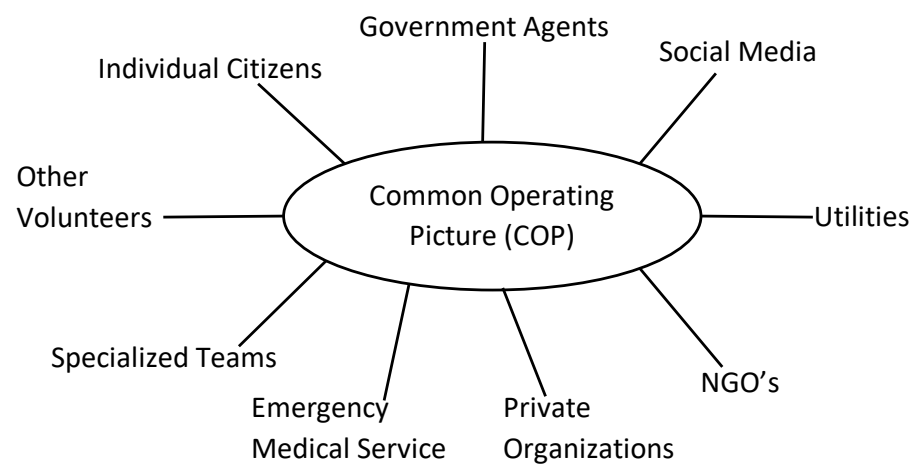

Figure 2: Common Operating Picture with various stakeholders

Extract, Load, and Transform (ETL) process. Then, obtain and queried by any reporting or visualization tools like Tableau.

\section{Case Study}

As a part of the Graduate Directed Project course, student teams implemented the CERT application by collecting and analyzing requirements from the client, a retired Brigadier General, US Army Reserve, who is also a professor in Emergency Disaster Management program at Northwest Missouri State University. The given case study is part of the project. Cognitive informatics is an interdisciplinary area that includes software engineering, psychology, and other subject areas. The requirements elicitation techniques such as interviewing and prototyping from the software engineering area is used to gather requirements. The details of the functional requirements are given in the Table. The project's goal is to implement a disaster management system for a wide variety of disasters and implement a common operating picture for different stakeholders, as shown in Fig. 2.

Stakeholders for a disaster include law enforcement officers and government agencies (municipal, county, state and federal), household utilities (electricity, natural gas, water supply, internet, and other telecommunications), Emergency Medical Services (hospitals, pharmacies, and ambulances), private organizations such as insurance, construction, and information technology companies, non-government organizations (red cross and handicap international). Several specialized teams such as firefighters, experts in diffusing radioactive materials and explosives, people with expertise in handling any toxic spills, oil, and chemical leaks. Resources such as pumper trucks, tanker trucks, aerial trucks, cranes, and life jackets. National and international volunteer groups, individual citizens, victims, and their families, social media are the essential stakeholders to be considered for an incident or a disaster.

Though the innovations of technology have increased, human life is still vulnerable to disasters. There are two kinds of disasters. They are natural disasters and human-made disasters. Examples of natural disasters are earthquakes, tornados, forest fires, etc. Any accidents, terrorism, biological warfare [1], including the use of toxins of biological origin or microorganisms as weapons of war, are a couple of examples of human-made disasters. For any disaster, there will be some common characteristics. They are the name of the accident, location (latitude and longitude), date and time, the number of living organisms injured or died, the number of people and days to complete the rescue, equipment, human and financial resources required for 


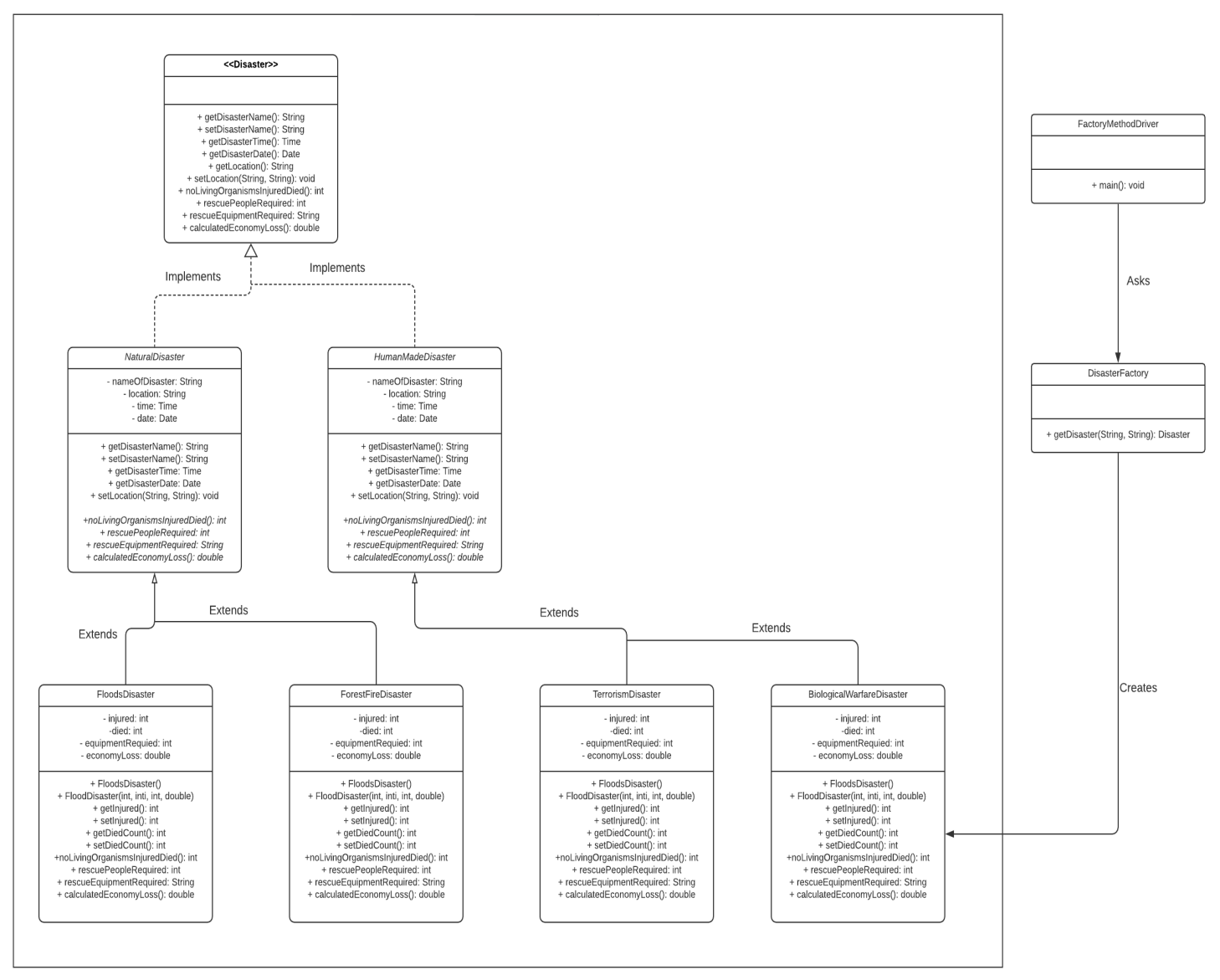

Figure 3: Factory method design pattern of EDMS

rescue operations, economic loss. Also, a disaster may have cascading psychological impacts of the people around the world, time to recover from the disaster itself, and many more. In such critical situations, businesses must communicate effectively to their customers, victims, and the rest of the world. One of the powerful ways of communication is updating their websites, mobile sites, and mobile apps. The user interfaces play a crucial role in displaying and bring up to date information to the audiences. Along with the websites' better usability, performance is also significant as the device users resist spending a considerable amount of time in panic situations.

Our CERT application is designed to accommodate a wide variety of disasters. Therefore, the design must be open for extensions and closed for modifications [2]. We used the factory pattern/factory method pattern to design the CERT software. A factory pattern defines an interface or an abstract class for creating an object, but the Java Virtual Machine (JVM) will decide which class to instantiate at run with some conditions. Here, we create an object without exposing the creation logic to the client and refer to a newly created object using a common interface. That means we can create objects for both natural and human-made disasters by hiding the implementation logic. If we need to add a new class, it is helpful to add without disturbing the existing class structure. The UML design of the factory pattern is shown in 
Table 1: Tasks list for usability testing

\begin{tabular}{|l|l|l|}
\hline Task ID & Task Description & User's Role \\
\hline T1 & Create incident report & Administrator \\
\hline T2 & Send the incident report & Rescue team member \\
\hline T3 & View preliminary information of the incident & Administrator \\
\hline T4 & Create a new resource & Administrator \\
\hline T5 & Assign resource for an incident & Administrator \\
\hline T6 & View incident on maps by different stakeholders & Rescue team member \\
\hline
\end{tabular}

Fig. 3.

We followed a systematic approach of usability testing with different roles (facilitator, two note-takers, and user). We used electronic prototypes for usability testing. We used tasks for the rescue team members and administrators. These tasks are related to assigning resources for an earthquake disaster and viewing the common operating picture for different stakeholders. Table 1 gives a summary of the tasks. All the users are the graduate students in applied computer science who are considered experts in using the system without training given about the system. The people who developed the prototypes are different from the users. We asked the users, both administrator and rescuer roles, to perform the tasks by the think-aloud process. The two note-takers record the observations of the user and the problems faced by them. We collected the data from ten different users on both roles. Then the data is analyzed and reported.

\section{Results}

The selected screenshots of the new EDMS are shown in Fig. 4. The initial version of these screen captures used for usability testing. The log keeper notes the design issues faced by the users while the users were performing usability testing. There were no significant issues for the users when performing tasks 1 through 5 . However, there were suggestions for better usage of colors, which will be addressed in our future research as we did not find any patterns from the users' opinions. The usage of colors in the user interfaces plays a vital role because we want to retain the user on the website or mobile app until the task has accomplished. A majority of users have faced issues in performing task T6. The problems were selecting the type of the stakeholder, limiting the data accesses to a few stakeholders, and filtering the historical data by triage codes. The access limitation of the dashboard to stakeholders is essential because of the security and anonymity of the data. A few data attributes are needed to be restricted to specific agencies for decision making.

\section{Conclusions and Future Work}

Using cognitive informatics principles, we extracted the users' knowledge and automated the resources' dynamic assignment at the disaster location. This helps to reduce the cognitive load of the rescuers at the workplace. We also proposed the architecture of COP with interactive dashboards of the comprehensive analytical EDMS and found a few design issues when various stakeholders access the dashboard. We accomplished this by conducting usability testing to find out the root causes of the problems faced by the users. The design issues were selecting the type of the stakeholder, limiting the data accesses to a few stakeholders, and filtering the historical data by triage codes. In the future, we focus on the usage of colors in the user 

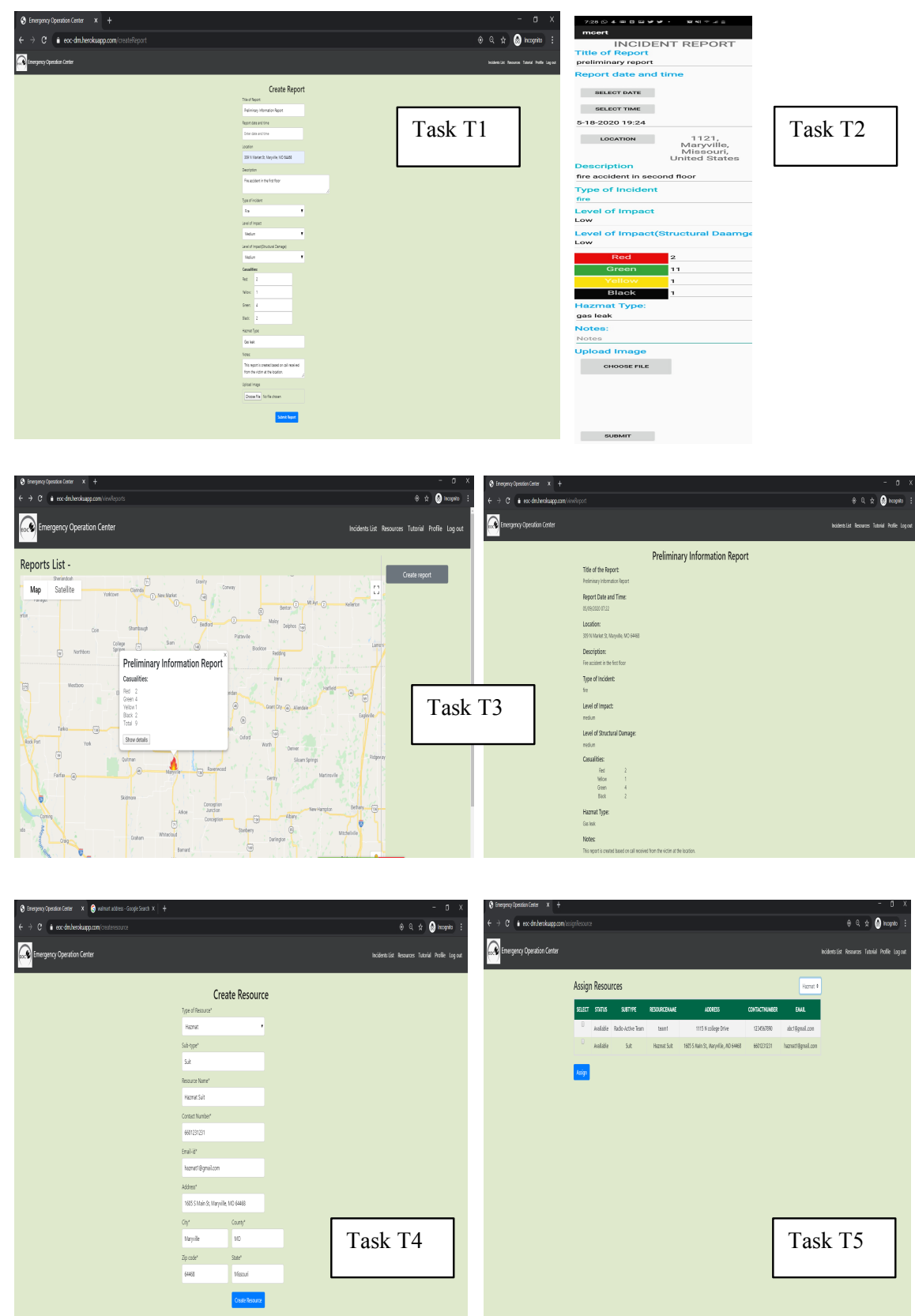

Figure 4: Screen captures of web and mobile application 
interfaces of EDMS. Also, on the issues of designing COP with interactive dashboard access to various stakeholders.

\section{Acknowledgements}

We thank Dr. Mark Corson for his service as a client for the graduate directed project course for several years and his insights on the project. We also acknowledge our student teams who developed this project as a part of their coursework.

\section{References}

[1] Keselman Alla, Laura Slaughter, and Vimla L. Patel. Toward a framework for understanding lay public's comprehension of disaster and bioterrorism information. In Biomedical Informatics, volume 38, pages 331-344, 2005.

[2] Ajay Bandi and Mark Corson. Designing an emergency management system using software design patterns. In Proceedings of 3rd International Conference on Electrical, Electronics, Engineering Trends, Communication Optimization and Sciences (EEECOS), pages 599-603, 2016.

[3] Ajay Bandi and Mark Corson. Design issues for converting websites to mobile sites and apps: A case study. In 2017 International Conference on Computing Methodologies and Communication (ICCMC), pages 652-656, 2017.

[4] Ajay Bandi, Aziz Fellah, and Harish Bondalapati. Mobile usability testing: Gathering evidence for designing user interfaces for emergency disaster management systems. In Proceedings of ISCA 28th International Conference on Software Engineering and Data Engineering, volume 38, 2018.

[5] Ajay Bandi and Phil Heeler. Usability testing: A software engineering perspective. In Proceedings:2013 International Conference on Human Computer Interaction (ICHCI), pages 1-8, 2013.

[6] Petr Kubíček and Karel Staněk. Dynamic visualization in emergency management. In Proceedings of First International Conference on Cartography and GIS, 2006.

[7] Kathleen Mastrian and Dee McGonigle. Cognitive informatics: An essential component of nursing technology design. In The Official Journal of American Academy of Nursing, volume 56, pages 332-333, 2008.

[8] Robin R. Murphy. Emergency informatics: Using computing to improve disaster management. In Computer, volume 49, pages 19-27, 2016.

[9] Pacific Northwest National Laboratory (PNNL). Learning and skill development. [online]. https: //www.pnnl.gov/cogInformatics/index.stm.

[10] The World Bank. Disaster risk management. [online]. https://www.worldbank.org/en/topic/ disasterriskmanagement/overview.

[11] YingXu Wang. On cognitive informatics. In Brain and Mind, volume 4, pages 151-167, 2003.

[12] Seungwon Yang, Haeyong Chung, Xiao Lin, Sunshin Lee, Liangzhe Chen, Andrew Wood, Andrea L. Kavanaugh, Steven D. Sheetz, Donald J. Shoemaker, , and Edward A. Fox. Phasevis: What, when, where, and who in visualizing the four phases of emergency management through the lens of social media. In Proceedings of the 10th International ISCRAM Conference, pages 912-917, 2013.

[13] Adam Zagorecki, Jozef Ristvej, Louis K. Comfort, and Tomas Lovecek. Executive dashboard systems for emergency management. In Communications-Scientific letters of the University of Zilina, volume 14, pages 82-89, 2012. 